\title{
Digital Compensation of High-performance Silicon Pressure Transducers
}

\author{
SELDEN B CRARY, WAYNE G BAER, JOHN C COWLES and KENSALL D WISE \\ Center for Integrated Sensors and Curcuts, Sold-State Electrontcs Laboratory, Unversuy of Michigan, \\ Ann Arbor, MI 48109-2122 (US A)
}

\begin{abstract}
Calibration and compensation have been performed on two silicon piezoresistive pressure transducers and a silicon capacitive pressure sensor, using a semi-automated, computer-based system Stepwise regression using polynomial functions is shown to be a useful technique for the calibration and compensation of these devices
\end{abstract}

\section{Introduction}

Testing and compensation represent very $1 \mathrm{~m}$ portant components of transducer cost today and are an important challenge in the development of next-generation devices aimed at the highaccuracy end of the applications spectrum For silicon pressure sensors, nonlinearity, offset and pressure sensitivity (slope) must be compensated with respect to both pressure and temperature Piezoresistive devices typically offer an uncompensated nonlinearity of about $05 \%$ of full scale ( 8 bits), while capacitive devices are more complex due to their inherent nonlinearity with pressure Both devices require substantial, but different, compensation The automated laser trimming of resistive compensation networks is typically used today to adjust the response of piezoresistive transducers, however, for piezoresistive-sensor accuracy above 8 bits, and for capacitive devices, improved compensation techniques are needed Digital compensation is an attractive approach because of the relative ease with which nonlinearities in the primary and secondary sensitivities can be handled, however, there is a vanety of possible approaches, extending from polynomials to segmented or full lookup tables Any technique selected should be cost effective, and both the memory requirements and the required test time vary considerably across the spectrum of possible approaches This paper reports research on the automatic digital polynomial-based compensation of silicon pressure transducers, with special attention paid to the bivariate compensation of these devices over pressure and temperature This work is aimed at the eventual realization of bus-organized sensors containing in-module compensation data and connected with a host processor as shown in Fig 1 [1] High performance here is generally defined as accuracy beyond the 8-bit level and is achieved by the application of techniques from response-surface methodology and multıdimensional regression

\section{Experimental}

Figure 2 shows the system used for data gathering and coefficient generation Within the test chamber, pressure and temperature can be controlled independently over the ranges 0 1000 Torr and $-40^{\circ} \mathrm{C}$ to $+180^{\circ} \mathrm{C}$, respectively For the devices studied (a commercial piezoresistive device, a device from our laboratory, and a capacitive device) characterization runs were made at fixed temperatures and pressures such that the deviations in sensor response at a given test point (after averaging multuple reads for noise suppression) were at a level of 13 bits (regarded as the system noise level) For each sensor, the functional form of the compensation function was determined by performing a stepwise regression [2] using the reference sensor response $(P)$ as the dependent vanable* and as many as 36 product terms, consisting of non-negative integer powers of the sensor response $R$ and the temperature $T$ in the form $R^{m} T^{n}$, where $0 \leqslant m \leqslant 5$ and $0 \leqslant n \leqslant 5$

\footnotetext{
*The choice of the reference pressure, rather than the sensor pressure, as dependent variable is motivated by the goal of obtaining a compensation formula, $P=P(R, T)$, into which can be entered the sensor response $R$ and the temperature Evaluation of this function provides the compensated pressure Care must be exercised to keep the bias, which is introduced by this particular form of calibration, within the bounds of accuracy required for the application at hand The issue of bias is discussed in ref 3
} 


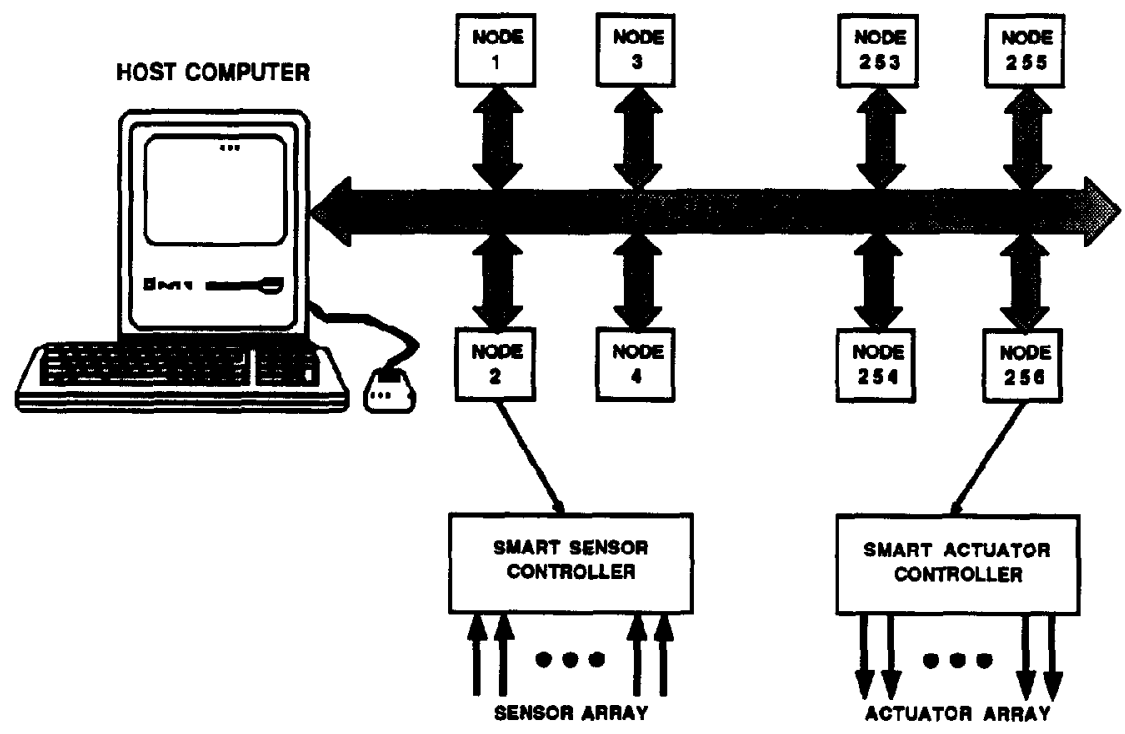

Fig 1 Organization of a multinode sensing system Each node has several sensors/actuators which are interfaced to the bidirectonal digital bus

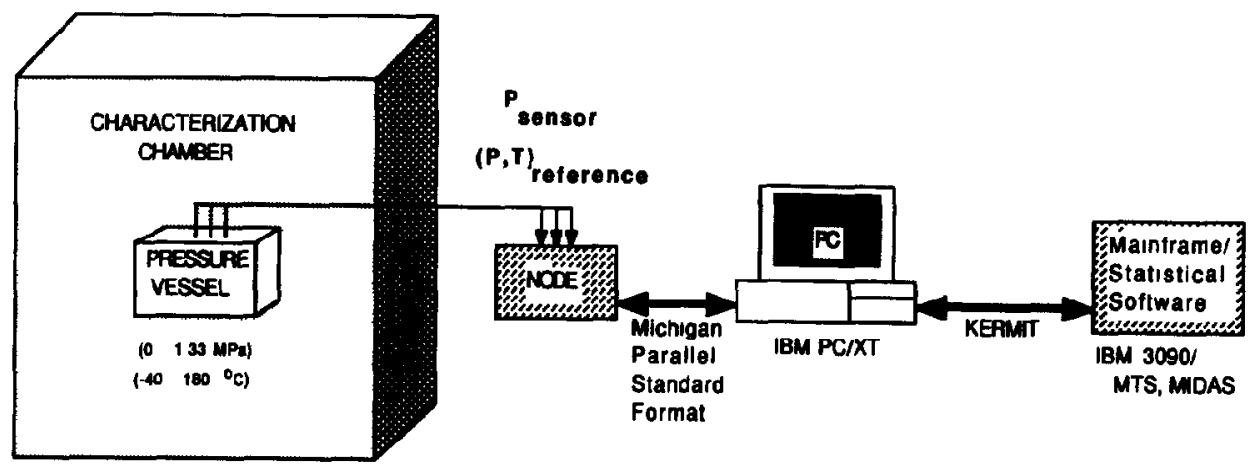

Fig 2 Automated characterization system for sensor compensation The system utilizes a bidirectional data bus as in Fig 1

\section{Results}

As an example, the piezoresistive sensor from our laboratory, which is composed of a polysilicon full bridge on a dielectrically coated thın (boron-stopped) silıcon membrane [4], was explored The device was fully characterized over 153 fixed $(P, T)$ data points, Fig 3 The response surface, after subtraction of the linear components of device response, is shown in Fig 4 The endpoint-to-endpoint nonlinearity, generalized to this bivanate case, was $29 \%$ FS, a value similar to that found with the commercial piezoresistive device After including nonlinear terms in the compensation, the nonlinearity was reduced by 44 bits, as shown in the histogram of the compensation error, $\mathrm{F}_{1 \mathrm{~g}} 5$, for 102 test points between $20^{\circ} \mathrm{C}$ and $120^{\circ} \mathrm{C}$ This device now offers a compensated standard error of 99 bits This result, and the others we have obtained, are tabulated in Table 1

TABLE 1 The standard errors ${ }^{a}$ expressed in bits, for the three devices $^{b}$

\begin{tabular}{ccccc}
\hline Sensor & Linear & Higher-order & $\begin{array}{l}\text { No of } \\
\text { terms }\end{array}$ & Improvement \\
\hline PZC (1D) & 84 & 11 & 6 & 26 \\
(2D) & 46 & 97 & 7 & 51 \\
PZM (1D) & 46 & 93 & 6 & 47 \\
(2D) & 55 & 99 & 14 & 44 \\
SCAP (2D) & 46 & 75 & 7 & 29 \\
\hline
\end{tabular}

Taken as $1 / 4$ of the $95 \%$ confidence interval

'PZC is a commercial piezoresistive device, PZM is the device from our laboratory [4], and SCAP is a silicon capacitive absolute-pressure device The column labeled 'linear' gives the standard error in compensation using only linear terms in the regressions

'From ref 1 


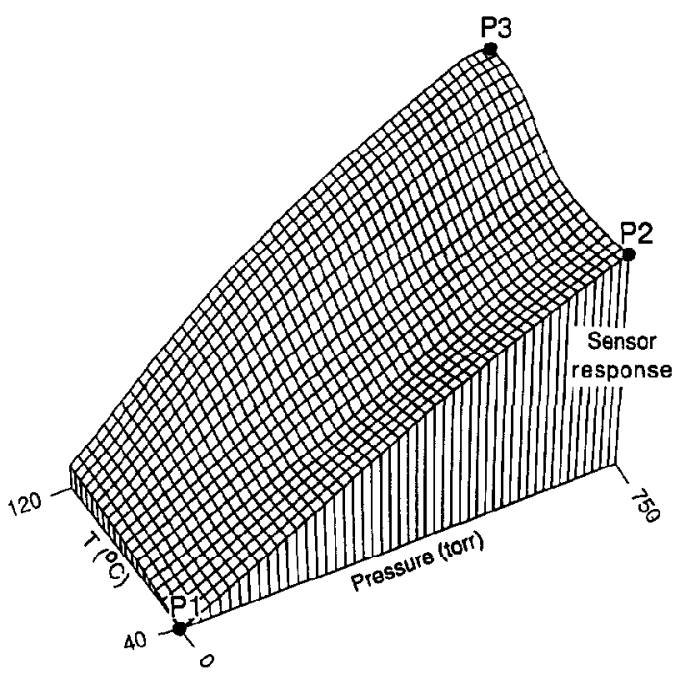

F1g 3 Response of the full-bridge device Three corner points of the domann of $(P, T)$ points are indicated These define a plane that can be used to determine the degree of nonlineanty of response The plane is the analog of the straight line between extreme endpoints often used as a measure of nonlinearity in univanate compensation

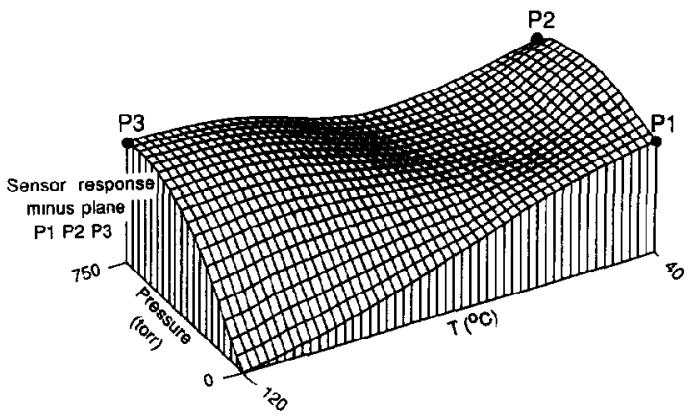

Fig 4 Nonlınear behavior of a full-bridge polysilicon piezoresistive pressure transducer over pressure and temperature The linear response has been subtracted from the measured data The view angle is different from that used in Fig 3

\section{Discussion}

The automated generation of the compensation polynomial is not significant in terms of test time, so that many devices could be tested in parallel in a production environment The techmique appears not only practical for commercial sensors but also allows research on the fundamental drift mechanisms in such devices at levels not previously reported

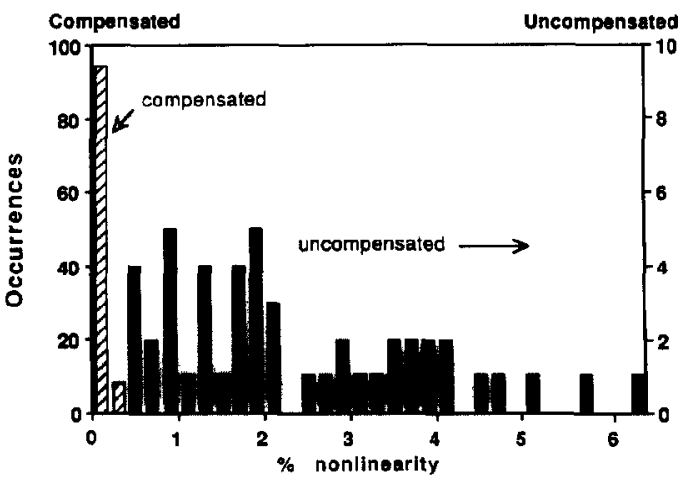

Fig 5 Histogram showing the distribution of occurrences of compensation error over the 102 data points for the polysilicon pressure transducer

Our ongoing work in the area of calibration and compensation is addressing the tradeoff between the number of test points and the resulting accuracy in order to define the minimum test time required for a given device and accuracy level We are also investigating the tradeoff between memory requirements and the computation time required for the evaluation of stored polynomials

\section{Conclusion}

We find that stepwise regression using polynomials provides a powerful technique for the compensation of silicon sensors, particularly for high-accuracy applications

\section{Acknowledgements}

This work has been supported by the Semiconductor Research Corporation under grant No 88MC-085 We appreciate helpful interactions with Joseph Grachino of the Ford Motor Company

\section{References}

I N Najafi, K W Clayton, W Baer, $\mathbf{K}$ Najafi and K D Wise, An architecture and interface for VLSI sensors, Tech Digest, IEEE Soltd-State Sensor Workshop, Hilton Head Island, SC, US A, June 6-9, 1988

2 N R Draper and H Smith, Applied Regression Analysis, Wiley, New York, 1966

$3 \mathrm{~J}$ Berkson, Are there two regressions?, $J$ Am Stat Assoc, 45 (1950) 164

$4 \mathrm{E}$ Yoon and K D Wise, A dielectrically-supported mult1element mass flow sensor, Digest of Papers, IEDM, San Franctsco, CA, US A, Dec 1988 